\title{
Distributed liquid level sensors using self-heated optical fibers for cryogenic liquid management
}

\author{
Tong Chen, ${ }^{1}$ Qingqing Wang, ${ }^{1}$ Rongzhang Chen, ${ }^{1}$ Botao Zhang, ${ }^{1}$ \\ Yuankun Lin, ${ }^{2}$ and Kevin P. Chen ${ }^{1, *}$ \\ ${ }^{1}$ Department of Electrical and Computer Engineering, University of Pittsburgh, Pittsburgh, Pennsylvania 15261, USA \\ ${ }^{2}$ Department of Physics, Electrical Engineering, University of North Texas, Denton, Texas 76203, USA \\ ${ }^{*}$ Corresponding author: pec9@ pitt.edu
}

Received 10 May 2012; revised 4 August 2012; accepted 6 August 2012;

posted 10 August 2012 (Doc. ID 168355); published 5 September 2012

\begin{abstract}
We present a continuous liquid level sensing system for both room temperature and cryogenic fluids with millimeter spatial resolution. Change of in-fiber Rayleigh backscattering signal from the distinct thermal response of the heated sensing fiber in liquid and in air were interrogated and spatially resolved using the optical frequency domain reflectometry. Both electrical and optical heating techniques were investigated for cryogenic liquid applications at $4 \mathrm{~K}, 77 \mathrm{~K}$, and the room temperature. The successful combination of self-heated fiber and wavelength-swept Rayleigh scattering interferometry provides, for the first time to our best knowledge, a truly distributed fuel gauge with high spatial resolution for cryogenic fuel storage, transportation, and management on ground and in space. (C) 2012 Optical Society of America
\end{abstract}

OCIS codes: $\quad 060.2370,060.4005,120.6810,120.5820$.

\section{Introduction}

Liquid level sensors are indispensible devices in fields such as chemical processing and fuel management on the ground and in space. They provide location information of liquid/gas interfaces to ensure safe and efficient liquid utilization. Historically, both mechanical and electrical liquid level sensors have been widely adopted and proved their reliability in practical usage for the room temperature environment. However, with the increase use of cryogenic liquid fuels such as liquefied natural gas and liquefied hydrogen in the economy, conventional liquid fuel gauges are finding their limitations in such cryogenic environments in terms of poor reliability and safety. Moreover, with the increasing complexity and scale of industry infrastructures to store, handle, and transport cryogenic liquid fuels, the number of liquid level sensors needed to track the distribution of

$1559-128 \mathrm{X} / 12 / 266282-08 \$ 15.00 / 0$

(C) 2012 Optical Society of America liquid fuel has risen rapidly. One of the best examples to exemplify this challenge is the need to track the distribution of cryogenic liquid fuel in microgravity environments. When the liquid is not bounded by the gravity, a large number of electrical sensors and wires are needed to gauge the volume and mass of liquid fuel for space applications.

In the recent 20 years, there has been a great deal of interest in developing fiber optic sensors for liquid level measurements. Fueled by the large demand in the telecommunications industry, compact and rugged fiber optic components are now commercially available at competitive prices. Fiber optic based sensors offer several key advantages over electrical sensors. They are immune to electromagnetic interference, spark-free, and chemically inert. A large numbers of sensors can be multiplexed on a single fiber feed through, and interrogated with one light source and detection unit. This technology greatly reduces the costs and implementation efforts for distributed multipoint sensing over complicated system. 
Liquid level sensors are usually divided into two categories: discrete level sensors and continuous level sensors. Discrete level sensors detect the liquid level at a single location and provide true/false information on whether the liquid level is above or below the sensing location. Numbers of discrete level sensors can be multiplexed at different positions on a single fiber to provide quasi-distributed sensing performance over long distance. But this capability is fundamentally limited by the multiplexing density of the discrete sensors and the consequently high manufacturing cost. On the other hand, continuous level sensors detect the liquid level in a certain range and provide quantitative information of the percentage that the liquid of interest has continuously occupied in the sensing range. This fully distributed sensing capability makes continuous level sensors a preferred solution for many mission-critical level sensing applications.

A majority of continuous fiber level sensors are based on the optical discrimination between refractive index (RI) of liquid and gas phases [1-6]. To measure the index difference between the liquid and gas, a number of schemes were used to exploit the evanescent wave sensing schemes. The sensing light in the single-mode fiber is partially exposed to the evanescent environment of the fiber surface. Due to the RI contrast between liquid and gas experienced by the sensing light, optical properties such as transmission loss, Bragg wavelength, and spectral interference wavelength can be interrogated upon data collection to determine the liquid level percentage in the sensing region. Using this approach, a number of level sensing methods have been reported, including long period grating [1], FBG with reduced cladding [2,3], sagnac fiber interferometer [4], multimode fiber interferometer [5], and a tapered fiber Mach-Zehnder interferometer [6]. Current RI-based continuous fiber level sensors are able to provide submillimeter spatial resolution with very fast response time. But the sensing distance is fundamentally limited by the length of the optical tap while the evanescent field is strong, which is typically no more than $100-200 \mathrm{~mm}$. The other limitation with this refractometric approach is that, for cryogenic fluids such as liquid hydrogen and liquid helium, their RI values are very close to their gaseous forms. This makes the RI discrimination impossible. An alternative sensing mechanism is needed for these low RI fluids. Guo et al. [7] reported a mechanical-optical method for continuous level sensing, in which the bending of cantilevers due to liquid occupation was interrogated using a FBG sensor. This approach provides precise continuous level sensing over extendable distance and temperature independence around room temperature, but its cryogenic temperature performance is susceptible to large thermal expansion mismatch between optical fiber and metal cantilevers over a wide temperature range.

Another important approach for liquid level sensing is through discrimination of thermal properties between liquid and gas. It has become a prime choice for fluid level sensing at cryogenic temperature. For example, the level of liquid hydrogen in cryogenic fuel tanks is monitored by a string of silicon diode point sensors. Excess electric current (tens of $\mathrm{mA}$; measuring current is $\sim 10 \mu \mathrm{A}$ ) is delivered to the local silicon sensor and heats it up. The voltage drop across the sensor is monitored to gauge the temperature rise. Due to the larger specific heat capacities and thermal convection rates of liquid hydrogen, the temperature rise in the liquid is much smaller than in the gas, which is used to determine the liquid level conditions. Thermal level sensing has also been employed with fiber optic sensors [8-11], in which the different heating responses of cryogenic liquid and gas were interrogated with multiplexed FBGs. However, the spatial resolution of the thermal-based level sensing approach is fundamentally limited by the number of point sensors deployed in the system. In both electronic and optical cases, the use of large numbers of point sensors will dramatically increase the cost and complexity of the system.

In this paper, we reported a continuous liquid level sensor for both room and low-temperature fluids with millimeter spatial resolution using self-heated fibers as sensing devices. It is based on the optical frequency domain reflectometry (OFDR) measurement of in-fiber Rayleigh scattering. This technology has been applied to provide a distributed sensing solution for in-fiber temperature, axial strain, pressure, gas flows, and hydrogen gas detection [12-17]. In this paper, both electrically and optically heated optical fibers are used to extend its application for liquid level sensing for both the room temperature and cryogenic environments down to $4 \mathrm{~K}$. Distinct heating response of liquid is discriminated from gas to measure the liquid level with millimeter spatial resolution. Level sensing operations were performed for pure water at room temperature, liquid nitrogen at $77 \mathrm{~K}$, and liquid helium at $4 \mathrm{~K}$. The sensing distance can be extended to more than $70 \mathrm{~m}$. The employment of commercially available metal-coated fiber and high attenuation fibers as the sensing device completely avoids the difficulties and high costs in manufacturing specialized fiber sensors such as fiber Bragg grating (FBG) with reduced cladding. The proposed sensing system provides a low-cost, long working distance, high resolution, and all-temperature solution to liquid level sensing applications.

\section{Sensing Principle}

The principle of in-fiber Rayleigh scattering measurement for distributed fiber sensing has been extensively reported [12-17]. It can be explained in brief using the OFDR diagram as illustrated in Fig. 1. The OFDR setup consists of the coherent tunable light source, the fiber interferometer, and the photodetector. Light from a CW laser is linearly swept and coupled into a fiber interferometer at frequency

$$
\omega(t)=\omega_{0}+\gamma t,
$$






Fig. 1. (Color online) Schematic of in-fiber Rayleigh backscattering measurement using optical frequency domain reflectometry.

where $\gamma$ is the wavelength tuning rate and $t$ is the laboratory time. The fiber interferometer is split into measurement and reference arms. The measurement arm of the interferometer is connected to the fiber under test (FUT) using a fiber recirculator. At the location $x$, the Rayleigh scattering signal and possible discrete reflections excited by the probe light in the FUT is reflected back to the OFDR system through the fiber recirculator and recombined with the reference arm light onto the photodetectors. The electrical field of the backscattering signal is expressed as

$$
E_{\text {Rayleigh }}=E_{\text {meas }}(t-\tau) \cdot \rho(\omega) \cos [\omega \tau-\phi],
$$

where $\tau$ is the time delay between reference arm path and measurement arm path at reflection location $x$, and $\rho$ and $\theta$ are the Rayleigh gain and phase shift. Upon the recombination, the light intensity measured by the photodetector can be expressed as

$$
\begin{aligned}
I(\omega)= & I_{\text {ref }}+I_{\text {Rayleigh }}+I_{\text {beat }} \\
= & \left|E_{\text {ref }}(t)\right|^{2}+\left|E_{\text {meas }}(t-\tau) \cdot r(\omega)\right|^{2}+2 E_{\text {ref }}(t) \\
& \cdot E_{\text {meas }}(t-\tau) \cdot \rho(\omega) \cos [\omega \tau-\phi],
\end{aligned}
$$

in which the location information of the Rayleigh backscattering is encoded into the beating terms. After the wavelength-swept measurement, this encoding can be retrieved through discrete Fourier transform upon the sweeping time/wavelength [12]

$$
I(\tau)=I(n x / c)=\operatorname{FFT}[I(\omega)]=\operatorname{FFT}\left[I_{\text {beat }}(\omega)\right]
$$

so that the Rayleigh scattering profile and possible discrete reflection information versus fiber length can be obtained. From the Fourier transform relation between time and spectral domain of measurement, the maximum probing length can be determined by the sampling rate of the OFDR, and the spatial resolution can be determined by the wavelength sweeping range of the coherent light source as

$$
L_{\max }=\frac{c \tau_{\text {sampling }}}{4 n}, \quad \Delta L=\frac{c}{2 n \Delta f_{\text {sweep }}} .
$$

It is also noted that the maximum probing length is fundamentally limited by the coherence length of the tunable laser.

When external perturbations such as temperature changes occur on certain FUT location, the pumping wavelength for Rayleigh scattering shifts with the induced local density change. By cross-correlating with a premeasured reference, spectral shifts of the Rayleigh signal can be spatially interrogated along the FUT $[\underline{13}, \underline{14}]$ as,

$$
I_{\text {measure }}(\omega) \otimes I_{\text {ref }}^{*}(\omega)=\operatorname{FFT}\left[I_{\text {measure }}(\tau) \cdot I_{\text {ref }}^{*}(\tau)\right],
$$

and these spectral shifts can be converted to temperature changes through the thermal-optic coefficient $K_{T}$ for the FUT,

$$
\frac{\Delta \lambda}{\lambda}=-\frac{\Delta \nu}{\nu}=K_{T} \Delta T
$$

\section{Liquid Level Sensing at Room Temperature Using Electrical On-fiber Heating}

Proof-of-concept liquid level sensing experiments were performed using deionized water at room temperature $\left(24^{\circ} \mathrm{C}\right)$. The experimental setup is illustrated in Fig. 2(a). A commercial OFDR unit from Luna Technologies (OBR 4600) was employed and butt-coupled with a piece of single-mode fiber with $20 \mu \mathrm{m}$ thick copper alloy coating (Oxford Electronics). Regulated current is applied to the metal surface of the fiber to generate resistive heating on the fiber, as shown on the right of Fig. 2(a). The heat is transferred into the fiber core and generates spectral shift of the Rayleigh signal. The OFDR unit consists of a tunable laser source from $1520-1610 \mathrm{~nm}$ and a pair of highly sensitive photodiodes with sampling rate of $2 \mathrm{MS} / \mathrm{s}$ which, according to (5), yields the spatial resolution of $\sim 10 \mu \mathrm{m}$ and maximum sensing distance of $\sim 70 \mathrm{~m}$ for Rayleigh signals. Scanning speed as high as $100 \mathrm{~nm} / \mathrm{s}$ can be employed to allow fast 


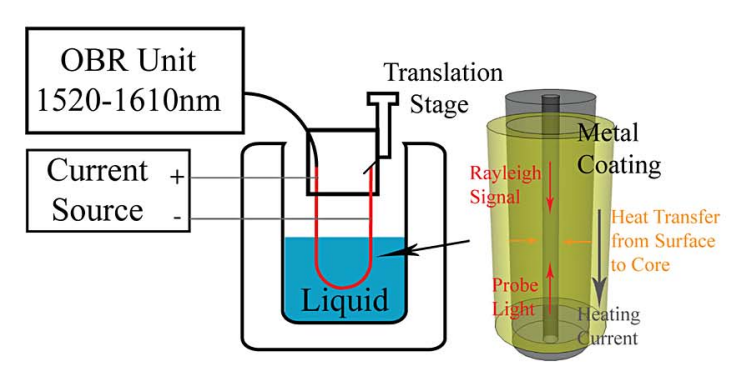

(a)

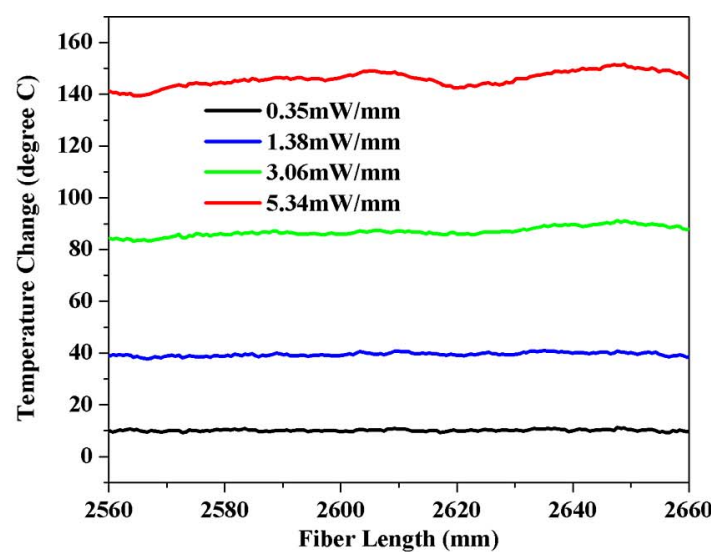

(b)



(c)

Fig. 2. (Color online) (a) Schematic of electrical on-fiber heating for liquid level sensing. (b) Uniform temperature profile using different heating power measured by in-fiber Rayleigh scattering. (c) Maximum temperature rise versus input electrical power density.

interrogation over the whole fiber length within seconds. The electrically heated fiber was folded and fixed with fiber holders on a translation stage and inserted into the deionized water. For each measurement point, the Rayleigh signal trace along the fiber is recorded with and without the on-fiber heating. A $1 \mathrm{~cm}$ long window was chosen to cross-correlate the two traces over a few tens of centimeters with submillimeter steps following Eq. (6). With Eq. (7), the resulting Rayleigh spectral shift trace is converted to on-fiber temperature profile. The absolute spectral and temperature accuracy obtained through the cross-correlation is inversely proportional to the size of the spatial windows. For conventional optical fiber with thermal-optic coefficient of $-0.8^{\circ} \mathrm{C} / \mathrm{GHz}$ or $12 \mathrm{pm} /{ }^{\circ} \mathrm{C}, 0.1^{\circ} \mathrm{C}$ accuracy in temperature change is expected with $1 \mathrm{~cm}$ long cross-correlating windows. On the other hand, thanks to the $10 \mu \mathrm{m}$ Rayleigh signal resolution and the fine cross-correlating steps, the practical spatial resolution for temperature profile is not strictly limited by the $1 \mathrm{~cm}$ window size if high temperature accuracy is not required. An abrupt temperature change of a few ${ }^{\circ} \mathrm{C}$ can be identified with submillimeter resolution.

Figures 2(b) and 2(c) show the electrical on-fiber heating results in air derived from OFDR Rayleigh measurements. The thermal-optic coefficient of $-1.6^{\circ} \mathrm{C} / \mathrm{GHz}$ is used for the copper-coated fiber [14], which is twice larger than the value for conventional Corning SMF-28 fiber. Due to a small thermal mass, a relatively low heating power of $0.35 \mathrm{~mW} / \mathrm{mm}$ (214 $\mathrm{mW}$ total) can uniformly heat up a section of $613 \mathrm{~mm}$ fiber by $10^{\circ} \mathrm{C}$, as shown in Fig. 2(b). When the input power on the fiber increases, the fluctuation in temperature profile becomes more pronouncing, which could be attributed to the coating imperfection and the influence of airflows. With $5.34 \mathrm{~mW} / \mathrm{m}$ input power, the fiber core is heated up by $145 \pm 5^{\circ} \mathrm{C}$. The linear dependence of temperature rise versus input heating power density is shown in Fig. 2(c), which demonstrates both the precision and flexibility in electrical on-fiber heating using metal-coated fiber.

When the heated fiber is dipped into water, abrupt temperature drops are observed in the heating profile, as shown in Fig. 3(a). This is because the on-fiber heat is taken away from the fiber surface into the surrounding water with heat transfer rate about 1000 times faster than into the air. This abrupt temperature gradient versus fiber position is interrogated by the in-fiber Rayleigh signal and serves as the indicator for liquid level position. The temperature profile of the folded sensing fiber at water level 


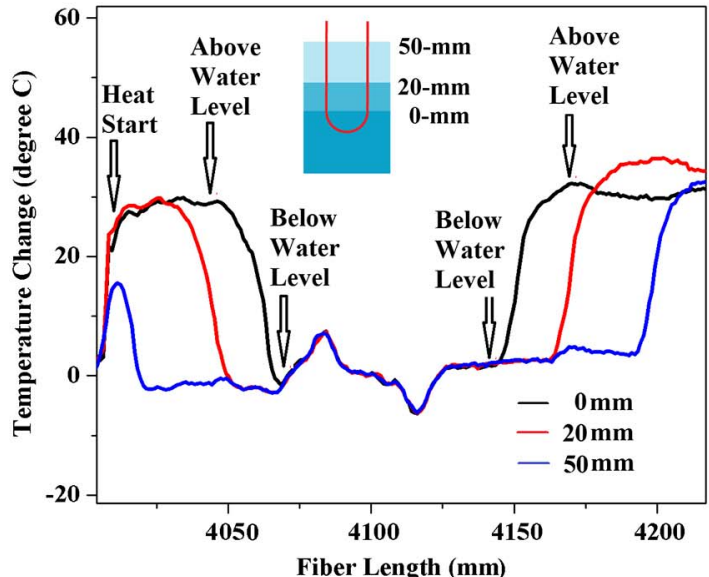

(a)

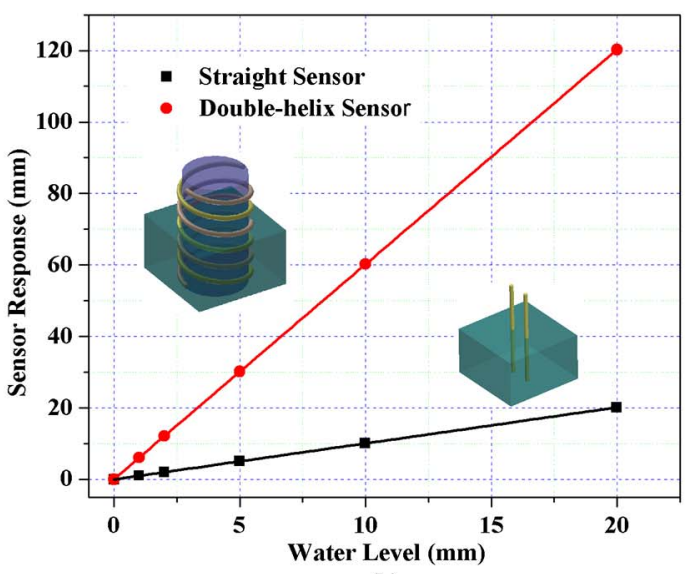

(b)

Fig. 3. (Color online) (a) Temperature profile of the electrically heated fiber during level sensing. (b) Sensor response of both straight and double-helix sensor configuration.

of 0,20 , and $50 \mathrm{~mm}$ (relative to the sensing fiber, reading from the micrometers on translation stage) are shown in Fig. 3(a).

Figure 3(b) shows the reliable operations of both straight and double-helix folded fiber sensors for water level from 0-20 mm. By winding the sensing fiber in two folds on the surface of a $1.5 \mathrm{in} .(38.1 \mathrm{~mm})$ diameter cylindrical post with $20 \mathrm{~mm}$ pitch, the level sensing resolution is enhanced by 6 times. Longer level sensing distance is available using extended metal-coated fiber. The maximum probing length for the OFDR unit we use is $\sim 70 \mathrm{~m}$, which could be further extended with higher sampling rate and better coherence control of the tunable laser.

\section{Measurement of Tilted Liquid Level}

In the preceding sections, water level sensing was performed using double-folded, self-heated fibers. For both the double-straight and double-helix configurations, two temperature abruptions can be observed in the heating profile at positions symmetric to the fiber folding point. This provides redundancy to the liquid level detection, which can be utilized to interrogate more degrees of freedom in level sensing. In a comprehensive liquid environment where acceleration force other than gravity is applied to the liquid body, the plane of liquid level can be tilted with respect to the level sensing system. To locate a tilted liquid level, three independent measurements, including the liquid level position at the center of the container $Z_{T}$, the tilting orientation $\theta_{T}$, and the tilting angle $\Phi_{T}$ needs to be interrogated, as is illustrated in Fig. 4(a). Theoretically, the three measurements can be uniquely determined by $>3$ data points of temperature abruption positions on the folded sensing fiber.

With only two data points [Abruption A and B, as shown in Fig. 4(a)] available for the double-helix configuration, we fixed the tilting orientation at $\theta_{T}=0$ and performed tilting level sensing for $\left(Z_{T}, \Phi_{T}\right)=$ $\left(0 \mathrm{~mm}, 0^{\circ}\right),\left(0 \mathrm{~mm}, 1^{\circ}\right),\left(0 \mathrm{~mm}, 5^{\circ}\right),\left(2 \mathrm{~mm}, 0^{\circ}\right)$, $\left(2 \mathrm{~mm}, 1^{\circ}\right)$, and $\left(2 \mathrm{~mm}, 5^{\circ}\right)$. A transfer matrix was



(a)

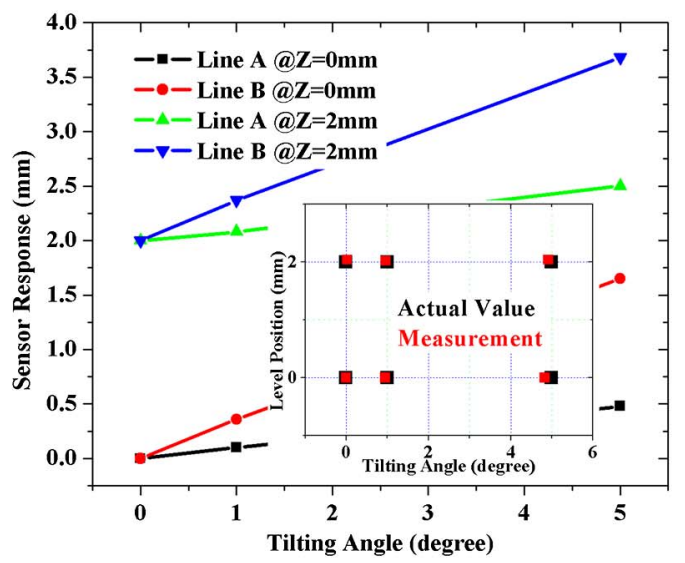

(b)

Fig. 4. (Color online) (a) Three independent parameters, the liquid level $Z_{T}$, the tilting orientation $\theta_{T}$, and the tilting angle $\Phi_{T}$ to determine a unique tilted liquid level. (b) The experimental results of measuring the liquid level $Z_{T}$ and tilting angle $\Phi_{T}$ using a double-helix sensor at fixed tilting orientation $\theta_{T}$. Inset: the comparison of actual value and measured value for liquid level $Z_{T}$ and tilting angle $\Phi_{T}$. 
derived to solve the liquid level and tilting angle from the two measured data points and the results are shown in Fig. 4(b). The solved values for the liquid level position and tilting orientation are compared with the actual values in the inset of Fig. 4(b) with good agreements. It is possible to fully determine the values of $\left(Z_{T}, \Phi_{T}, \theta_{T}\right)$ if a triple-helix sensing configuration is implemented with three thermal abruption data points.

\section{Level Sensing for Cryogenic Fluids with Optical In- fiber Heating}

In the previous sections, we have applied electrical current to the metal coating of optical fiber for onfiber heating. The electrical heating scheme provides a low-cost and energy efficient way to heat on-fiber sensors with uniform temperature profiles. However, this approach is vulnerable to a number of harsh environments. First, the exposed electrical current presents a spark hazard in the case of flammable liquid applications, although this risk can be mitigated through additional fiber packaging. This limits its application in highly volatile liquid fuel management. Second, although the metal coating serves as a good protection to the optical fiber for impact, stretching, and extreme temperature fluctuation, the coating is vulnerable to corrosive chemicals, which presents another limitation to the implementation of the level sensing method. Furthermore, in extreme low temperature environments such as in liquid hydrogen at $10 \mathrm{~K}$ and liquid helium at $4 \mathrm{~K}$, the resistivity of metal decreases dramatically; thus, large electrical currents are needed to heat up the fiber.

Some of these disadvantages can be circumvented through fiber packaging techniques, but other disadvantages are inherent, which can only be solved by the use of other on-fiber heating approaches. In this section, we explore the application of on-fiber optical heating schemes for cryogenic liquid level sensing. While the proliferating use of cryogenic liquid fuel in our economy demands more reliable and sensitive sensing technology, cryogenic environments are among the most challenging environments to perform sensing tasks.

On-fiber optical heating has generated wide interest for active fiber sensing applications due to its coherence with the optical fibers [8-11]. The heating power is delivered optically through the optical fiber

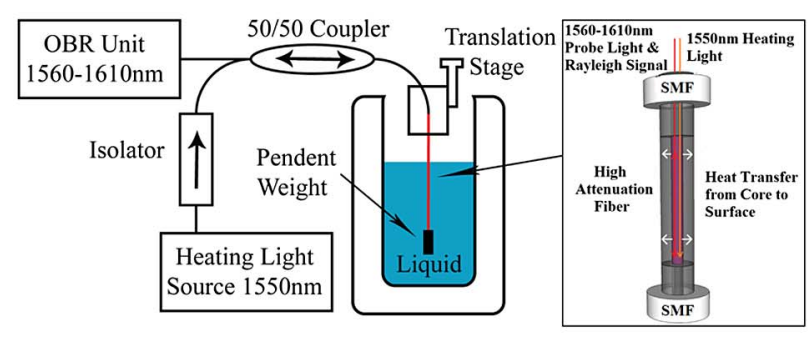

(a)

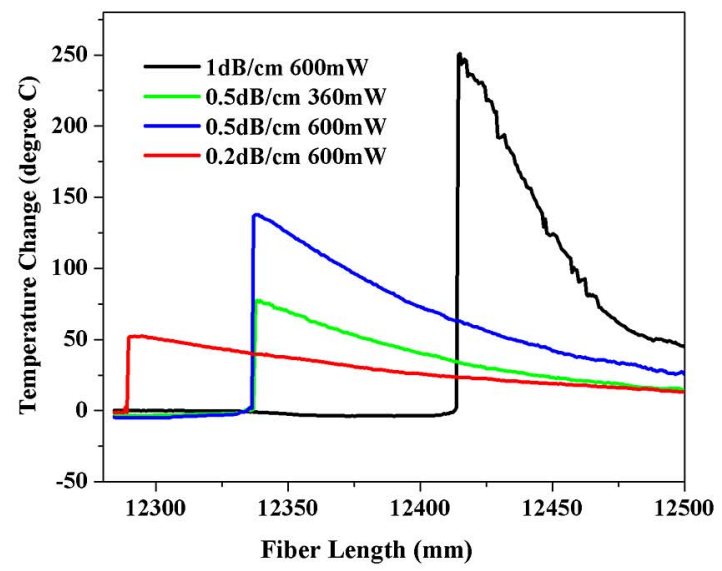

(b)

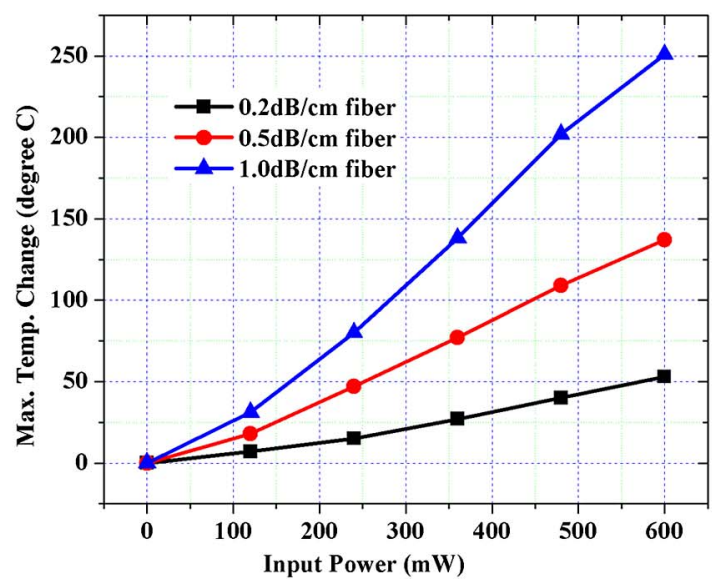

(c)

Fig. 5. (Color online) (a) Schematic of level sensing using optically heated fiber. (b) Temperature profiles of different HAF with different heating power. (c) Heating efficiency of different HAF. 


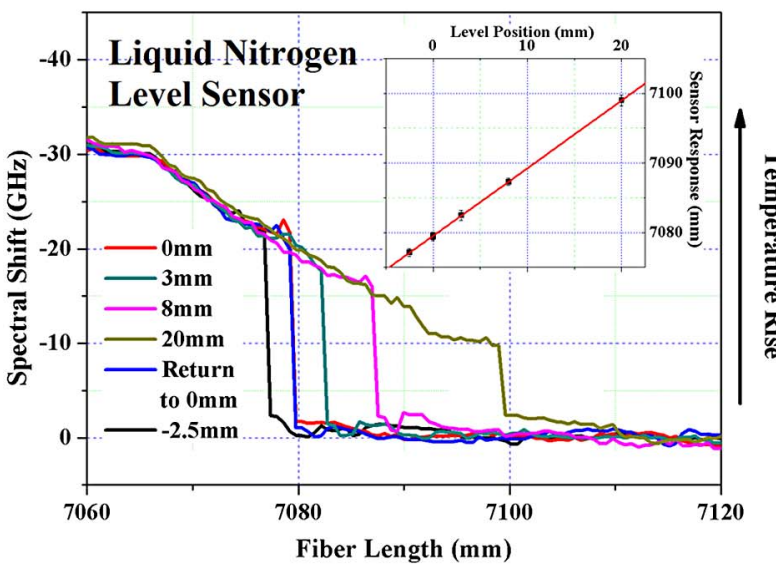

(a)



(b)

Fig. 6. (Color online) Level sensing response in (a) liquid nitrogen (LN) at $77 \mathrm{~K}$ and (b) liquid helium (LH) at $4 \mathrm{~K}$.

and released to the designated on-fiber sensing region through optical taps. Different optical heating techniques were developed using multimode fibers [8], and more recently, single-mode high attenuation fibers (HAF) [9-11,16]. Compared with the multimode fiber optical delivery schemes [8], optical taps using single-mode high attenuation fibers offers precise control over optical heating and optical power distribution. This approach is used in this paper for cryogenic liquid level sensing at $4 \mathrm{~K}$ and $77 \mathrm{~K}$, respectively.

The schematic of optically heated fiber level sensors is illustrated in Fig. 5(a). Light from a CW laser at $1550 \mathrm{~nm}$ was amplified up to $2 \mathrm{~W}$ using an Erbium-doped fiber amplifier as the heating light source. The heating light is combined with the tunable probe light at $1560-1610 \mathrm{~nm}$ from the OFDR unit using a bidirectional 50:50 fiber coupler and injected into a piece of $\mathrm{HAF}$ with $1 \mathrm{~dB} / \mathrm{cm}$ loss in the single-mode fiber core. Optical power is attenuated and transformed into the heating power in the fiber core, which is used to heat up the entire fibers. The in-fiber heating profiles of HAFs with $0.2,0.5$, and $1 \mathrm{~dB} / \mathrm{cm}$ attenuation coefficient at different optical heating powers are interrogated with spatially resolved Rayleigh signal in the air. Based on previous works on FBG sensors written in attenuation fiber $[9,18]$, the thermal-optical coefficient of $12 \mathrm{pm} /{ }^{\circ} \mathrm{C}$ was determined for attenuation fibers around room temperature and used here to calculate the temperature profile. The results are shown in Fig. 5(b) and 5(c). Contrary to the uniform heating profile of electrically heated fiber, exponential decays of temperature are observed due to the core attenuation. With $600 \mathrm{~mW}$ input power, $1 \mathrm{~dB} / \mathrm{cm}$ loss $\mathrm{HAF}$ can be heated up to $251^{\circ} \mathrm{C}$ at maximum. With a less lossy $\mathrm{HAF}$ of $0.2 \mathrm{~dB} / \mathrm{cm}$, the fiber can be heated up by $30-50^{\circ} \mathrm{C}$ in a $30 \mathrm{~cm}$ long region.

For cryogenic liquid level measurement, the HAF is suspended from a translation stage down to the cryogenic dewar. The fiber is kept straight using a $5 \mathrm{~g}$ pendent weight. When the optical heating is turned off, the temperature gradient around the liquid level is measured as the reference. With the optical power turning on, the temperature profile along the fiber is measured again and compared with the reference. Because the thermal-optic coefficient of HAF at cryogenic temperature is unknown, we use the Rayleigh spectral shift values instead of the absolute temperature to describe the measured temperature changes. The level sensing results for liquid nitrogen at $77 \mathrm{~K}$ and liquid helium at $4 \mathrm{~K}$ are shown in Fig. 6(a) and $6(\mathrm{~b})$, respectively. For both liquids, the temperature changes at liquid levels are steeper than the case for electrically heated level sensor. The abruption width is as thin as $\sim 1 \mathrm{~mm}$ for heated HAF due to both the inside-out heating approach and the elimination of thick metal coating. The optically heated HAF level sensor demonstrates higher spatial resolution but shorter sensing range compared to the electrically heated metal coated sensor due to the large optical attenuation. This problem can be potentially mitigated using a HAF fiber with low optical attenuation. For level sensing applications, a $1-5^{\circ} \mathrm{C}$ temperature drop is sufficient to distinguish the level. This allows a much longer sensing fiber heated by limited optical power fed into the HAF.

\section{Conclusions}

In summary, we reported for the first time, to our best knowledge, a continuous and distributed liquid level sensing system based on the Rayleigh scattering measurement of active fiber. By scanning self-heated fiber with a tunable laser in OFDR configuration, the temperature abruptions at the liquid/ gas interfaces are identified along the fiber to provide fast and reliable detection of liquid distribution. The technology demonstrated submillimeter spatial sensing resolution for a sensing range up to $70 \mathrm{~m}$ in a wide temperature range down to $4 \mathrm{~K}$ for cryogenic liquids. It is also capable of detecting comprehensive liquid level distributions in microgravity and accelerating environments, which would be uniquely attractive to space science applications. The simplicity and wide applicability of the technique make it one of the 
most versatile solutions to liquid level sensing applications for a wide range of harsh environments.

This work was supported by the National Science Foundation (CMMI-0644681, CMMI-0900564, CMMI-1109971) and the Department of Energy (DE-FE0003859).

\section{References}

1. S. Khahiq, S. W. James, and R. P. Tatam, "Fiber-optic liquid-level sensor using a long-period grating," Opt. Lett. 26, 1224-1226 (2001).

2. B. Yun, N. Chen, and Y. Cui, "Highly sensitive liquid-level sensor based on etched fiber Bragg grating," IEEE Photon. Technol. Lett. 19, 1747-1749 (2007).

3. S. M. Chandani and N. A. F. Jaeger, "Optical fiber-based liquid level sensor," Opt. Eng. 46, 114401 (2007).

4. B. Dong, Q. Zhao, L. Feng, T. Guo, L. Xue, S. Dong, and H. Gu, "Liquid-level sensor with a high-birefringence-fiber loop mirror," Appl. Opt. 45, 7767-7771 (2006).

5. J. E. Antonio-Lopez, J. J. Sanchez-Mondragon, P. LiKam Wa, and D. A. May-Arrioja, "Fiber-optic sensor for liquid level measurement," Opt. Lett. 36, 3425-3427 (2011).

6. T. Chen, R. Chen, P. Lu, Q. Chen, and K. P. Chen, "Fiber MachZehnder Interferometer for Liquid Level Sensing," Electron. Lett. 47, 1093-1095 (2011).

7. T. Guo, Q. Zhao, Q. Dou, H. Zhang, L. Xue, G. Huang, and X. Dong, "Temperature-insensitive fiber Bragg grating liquidlevel sensors based on bending cantilever beam," IEEE Photon. Technol. Lett. 17, 2400-2402 (2005).

8. K. P. Chen, B. McMillan, and L. Cashdollar, "Self-heated fiber Bragg grating sensors," Appl. Phys. Lett. 86, 143503 (2005).
9. T. Chen, D. Xu, M. Buric, M. Maklad, P. R. Swinhart, and K. P. Chen, "Self-heated all-fiber sensing system for cryogenic environments," Meas. Sci. Technol. 21, 094036 (2010).

10. F. Yei, T. Chen, D. Xu, K. P. Chen, and L. Qian, "Cryogenic fluid level sensor multiplexed by frequency-shifted interferometry," Appl. Opt. 49, 4898-4905 (2010).

11. T. Chen, M. Maklad, P. R. Swinhart, and K. P. Chen, "Self-heated optical fiber sensor array for cryogenic fluid level sensing," IEEE Sens. J. 11, 1051-1052 (2011).

12. B. Soller, D. Gifford, M. Wolfe, and M. Froggatt, "High resolution optical frequency domain reflectometry for characterization of components and assemblies," Opt. Express 13, 666-674 (2005).

13. S. T. Kreger, D. K. Gifford, M. E. Froggatt, B. J. Soller, and M. S. Wolfe, "High resolution distributed strain or temperature measurements in single- and multimode fiber using swept-wavelengh interferometry," in Optical Fiber Sensors (Optical Society of America, 2006), paper ThE42.

14. A. K. Sang, M. E. Froggatt, D. K. Gifford, S. T. Kreger, and B. D. Dickerson, "One centimeter spatial resolution temperature measurements in a nuclear reactor using Rayleigh scatter in optical fiber," IEEE Sens. J. 8, 1375-1380 (2008).

15. T. Chen, Q. Wang, R. Chen, B. Zhang, C. Jewart, K. P. Chen, M. Maklad, and P. R. Swinehart, "Distributed high temperature pressure sensing using air-hole microstructural fibers," Opt. Lett. 37, 1064-1066 (2012).

16. T. Chen, Q. Wang, R. Chen, B. Zhang, and K. P. Chen, "Distributed flow sensing using hot optical wire grids," Opt. Express 20, 8240-8249 (2012).

17. T. Chen, Q. Wang, R. Chen, B. Zhang, K. P. Chen, M. Maklad, and P. R. Swinehart, "Distributed hydrogen sensing using infiber Rayleigh scattering," Appl. Phys. Lett. 100, 191105 (2012).

18. S. Gao, A. P. Zhang, H-Y. Tam, L. H. Cho, and C. Lu, "Alloptical fiber anemometer based on laser heated fiber Bragg gratings," Opt. Express 19, 10124-10130 (2011). 